\title{
The family history score tool identifies high risk families for colorectal cancer
}

\author{
Janet Shenal ${ }^{1,2^{*}}$, Ellen McGannon ${ }^{1,2}$, Susan Fay ${ }^{1,2}$, Loretta Arroyo ${ }^{1,2}$, James M Church ${ }^{2}$ \\ From 13th Annual Meeting of the Collaborative Group of the Americas on Inherited Colorectal Cancer \\ Honolulu, Hawaii, USA. 16-17 October 2009
}

\section{Background}

Assessing family history risk of colorectal cancer (CRC) is often difficult due to complex permutations and combinations. A family history score has been developed and validated to simplify familial risk assessment. This study examines the usefulness of this scoring system to identify families at risk through public outreach programs.

\section{Methods}

A published Family History Scoring System ${ }^{1}$ was used to determine familial cancer risk at 7 health fair and educational events spanning 2006-2008. Patients with scores $\leq 7$ are considered at moderate risk for advanced adenomas or CRC, scores with $>7$ signify high risk, and scores $>10$ are suggestive of a hereditary colorectal cancer syndrome. A computer program using touch screen technology was designed to record and calculate family history score and was used at the women's and men's health fairs. No identifying information was collected.
Recommendations for surveillance were given depending on the risk level assigned.

\section{Results}

1223 people participated. The scores are summarized in Table 1.

Author details

${ }^{1}$ Taussig Cancer Institute, Cleveland Clinic, Cleveland, Ohio 44195, USA. ${ }^{2}$ The Sanford R. Weiss, M.D. Center for Hereditary Colorectal Neoplasia, Cleveland Clinic, Cleveland, Ohio 44195, USA.

Published: 25 May 2010

Reference

1. Church JM: Family History Scoring System. Dis Col Rectum 2005, 48:889-96

doi:10.1186/1897-4287-8-S1-P21

Cite this article as: Shenal et al:: The family history score tool identifies high risk families for colorectal cancer. Hereditary Cancer in Clinical Practice 2010 8(Suppl 1):P21.

Table 1

\begin{tabular}{|c|c|c|c|c|c|c|c|c|c|}
\hline \multirow[t]{2}{*}{ Screening Event/Year } & \multicolumn{8}{|c|}{ Scoring Range } & \multirow[t]{2}{*}{ Tota } \\
\hline & $\begin{array}{c}0 \\
\text { Avg Risk }\end{array}$ & $\%$ & $\begin{array}{c}1-7 \\
\text { Mod Risk }\end{array}$ & $\%$ & $\begin{array}{c}\text { 8-10 } \\
\text { High Risk }\end{array}$ & $\%$ & $\begin{array}{c}>10 \\
\text { High Risk }\end{array}$ & $\%$ & \\
\hline Minority Health Fair '06 & 114 & 83 & 23 & 17 & 0 & & & & 137 \\
\hline Women's Health Fair '06 & 203 & 75 & 63 & 23 & 1 & 1 & 3 & 1 & 270 \\
\hline Minority Health Fair '07 & 130 & 83 & 23 & 14 & 3 & 2 & 1 & 1 & 157 \\
\hline Women's Health Fair '07 & 128 & 73 & 41 & 23 & 3 & 2 & 3 & 2 & 174 \\
\hline Minority Health Fair '08 & 66 & 86 & 10 & 13 & 0 & 0 & 1 & 1 & 77 \\
\hline CRC Awareness Month '08 & 116 & 47 & 115 & 46 & 7 & 3 & 10 & 4 & 248 \\
\hline Women's Health Fair '08 & 116 & 73 & 33 & 21 & 9 & 5 & 1 & 1 & 159 \\
\hline Total & 873 & 71 & 308 & 25 & 23 & 2 & 19 & 2 & 1123 \\
\hline
\end{tabular}

\footnotetext{
* Correspondence: shenalj@ccf.org

${ }^{1}$ Taussig Cancer Institute, Cleveland Clinic, Cleveland, Ohio 44195, USA
} 